\title{
RANDOM FIXED POINT THEOREMS IN BANACH ALGEBRAS WITH APPLICATIONS TO RANDOM INTEGRAL EQUATIONS
}

\author{
B. C. DHAGE
}

\begin{abstract}
The present paper studies the random versions of some deterministic fixed point theorems of Dhage [5] and Dhage and Regon [7]. Applications are given to a certain nonlinear functional random integral equation for proving the existence of random solution under the generalized Lipschitzicity and Caratheodory conditions.
\end{abstract}

\section{Introduction}

Random fixed point theorems are the stochastic generalizations of the classical or deterministic fixed point theorems in abstract spaces and are useful in the study of nonlinear random equations for proving the existence and uniqueness theorems. Spacek [18] and Hans [9] have proved the stochastic analoge of Banach fixed point theorem in a seperable metric space and Mukherjea [16] proved the random version of Schauder's fixed point theorem on an atomic probability measure space. Then in [17], Prakash Rao has obtained the random version of the second important topological fixed point theorem of Krasnoselskii [12] on an atomic probability measure space. Further Itoh in [11] has generalized all these random fixed point theorems in a separable metric space. The details appear in Papageorgiou [15]. In this paper we obtain a random version of a third important topological fixed point theorem of Dhage [5] in a seperable Banach algebra via the method of mesurable selectors and apply it to a nonlinear functional random integral equation of mixed type for proving the existence result. We need the following results on measureability and measurable selectors for the multi-valued mappings given by Himmelberg [10] and Kuratowskii and Ryll-Nardzewski [14] in the sequel.

Theorem 1.1. [9] Let $(\Omega, \mathcal{A})$ be a measurable space and let $X$ be a seperable Banach space with a $\sigma$-algebra $\beta_{X}$ of all Borel subsets of $X$. If the multi-valued map $F: \Omega \rightarrow 2^{X}$ has closed values i.e. $F(\omega)$ is closed for each $\omega \in \Omega$, then the following statements are equivalent.

(a) $F$ is measurable

(b) $\omega \rightarrow d(x, F(\omega))$ is measurable for each $x \in X$.

Received October 15, 2001; revised May 9, 2002.

2000 Mathematics Subject Classification. 47H10.

Key words and phrases. Random fixed point theorem, random integral equation. 
(c) $\operatorname{Gr}(F)$ is $\left(\mathcal{A} \times \beta_{X}\right)$-measurable, where $\operatorname{Gr}(F)$ is the graph of the multi-valued map $F$.

Theorem 1.2. [13] If $F: \Omega \rightarrow 2^{X}$ is a measurable multi-valued map with closed values, then $F$ has a measurable selector.

In the following section we give some prelimineries needed in the sequel.

\section{Prelimineries}

Throughout this paper, let $(\Omega, \mathcal{A})$ denote a measurable space, $X$ a Banach space with norm $\|\cdot\|$ and let $\beta_{X}$ denote the $\sigma$-algebra of all Borel subsets of $X$. Let $2^{X}, C L(X)$, $B(X), C(X), K(X), C B(X)$ and $K C(X)$ denote respectively the families of non-empty, closed, bounded, convex, closed and bounded, and the compact-convex subsets of $X$. Following Himmelberg [10], a multi-valued mapping $F: \Omega \rightarrow 2^{X}$ is called measurable if for any open subsets $G$ of $X$,

$$
F^{-1}(G)=\{\omega \in \Omega \mid F(\omega) \cap G \neq \phi\} \in \mathcal{A} .
$$

Notice that when $F(\omega) \in K(X)$ for all $\omega \in \Omega$, then $F$ is measurable if and only if $F^{-1}(C) \in \mathcal{A}$ for every closed subset of $C$ of $X$. See Himmelberg [10]. A measurable mapping $\xi: \Omega \rightarrow X$ is called a measurable selector of the multi-valued map $F$ if $\xi(\omega) \in$ $F(\omega)$ for all $\omega \in \Omega$. A mapping $T: \Omega \times X \rightarrow X$ is called a random operator if $T(\cdot, x)$ is measurable for all $x \in X$ and generally expressed as $T(\omega, x):=T(\omega) x$. A measurable mapping $\xi: \Omega \rightarrow X$ is called a random fixed point of the random operator $T: \Omega \times X \rightarrow X$ if $T(\omega, \xi(\omega))=T(\omega) \xi(\omega)=\xi(\omega)$ for every $\omega \in \Omega$.

Let $T: X \rightarrow X$. Then $T$ is called compact if $\overline{T(X)}$ is a compact subset of $X$. Again $T$ is called totally bounded if for any bounded subset $S$ of $X, T(S)$ is a totally bounded or precompact subset of $X$. Finally $T$ is called completely continuous if it is continuous and totally bounded on $X$. Similarly a random operator $T(\omega): \Omega \times X \rightarrow X$ is called compact (resp. totally bounded and completely continuous) if the operator $T(\omega)$ is compact (resp. totally bounded and completely continuous) for each $\omega \in \Omega$. Note that every compact operator (random operator) is totally bounded operator (random operator) on $X$, but the converse may not be true. However two notions are equivalent on a bounded subset of $X$.

Again a mapping $T: X \rightarrow X$ is called $D$-Lipschitzician if there exists a continuous nondecreasing function $\phi: \boldsymbol{R}^{+} \rightarrow \boldsymbol{R}^{+}$such that

$$
\|T x-T y\| \leq \phi(\|x-y\|)
$$

for all $x, y \in X$, where $\phi(0)=0$. Similarly a random operator $T(\omega): \Omega \times X \rightarrow X$ is called $D$-Lipschitzician if there exists a function $\phi: \Omega \times \boldsymbol{R}^{+} \rightarrow \boldsymbol{R}^{+}$such that for each $\omega \in \Omega$,

$$
\|T(\omega) x-T(\omega) y\| \leq \phi_{\omega}(\|x-y\|)
$$


for all $x, y \in X$, where $\phi_{\omega}(r)=\phi(\omega, r)$ with $\phi(\omega, 0)=0$ for all $\omega \in \Omega$.

If $\phi_{\omega}(r)=\alpha(\omega) r, \alpha(\omega)$ being a positive real-valued function on $\Omega$, then $T(\omega)$ is called a Lipschitzician with Lipschitz constant $\alpha(\omega)$. Futher if $\alpha(\omega)=1 \forall \omega \in \Omega$, the random operator $T(\omega)$ is called nonexpansive and when $\alpha(\omega)<1 \forall \omega \in \Omega, T(\omega)$ is called a contraction with a contraction constant $\alpha(\omega)$. Finally if $\phi_{\omega}(r)<r, r>0$ for each $\omega \in \Omega$, where $\phi$ is not necessarily nondecreasing, then the random operator $T(\omega)$ is called a nonlinear contraction. In the special case when the random parameter $\omega$ is absent, we obtain the operator $T$ to be a deterministic nonlinear contraction on $X$. See Boyd and Wong [3].

The measure of noncompactness of a bounded set $S$ in $X$ is a nonnegative real number $\alpha(S)$ defined by

$$
\alpha(S)=\inf \left\{r>0: S=\bigcup_{i=1}^{n} S_{i}, \operatorname{diam}\left(S_{i}\right) \leq r, \forall i\right\} .
$$

The above measure $\alpha$ of noncompactness is due to Kuratowskii and the details may be found in Banas and Goebel [1]. A mapping $T: X \rightarrow X$ is called condensing if for any bounded set $S$ of $X, T(S)$ is bounded and $\alpha(T(S))<\alpha(S)$ for $\alpha(S)>0$. Similarly a random operator $T(\omega): \Omega \times X \rightarrow X$ is called condensing if the operator $T(\omega)$ is condensing for each $\omega \in \Omega$.

In the present paper, we prove the random version of the following fixed point theorem of Dhage [5].

Theorem 2.1. Let $S$ be a non-empty, closed, convex and bounded subset of a Banach algebra $X$ and let $A, B: S \rightarrow X$ be two operators such that

(a) A is D-Lipschitzician,

(b) $B$ is completely continuous, and

(c) $A x B x \in S$ for each $X \in S$.

Then the operator equation

$$
A x B x=x
$$

has a solution in $S$, whenever $M \phi(r)<r, r>0$, where $M=\|B(S)\|=\sup \{\|B x\|: x \in S\}$.

\section{Random Fixed Point Theory}

We need the following two useful lemmas in the sequel.

Lemma 3.1. Let $S \subset X$ and let $A, B: S \rightarrow X$ be two operators satisfying

(a) A is D-Lipschitzician, and

(b) B is continuous and compact. 
Then the mapping $T: S \rightarrow X$ defined by $T x=A x B x$ is continuous.

Proof. Let $\left\{x_{n}\right\}$ be a sequence in $S$ converging to a point $x \in S$. Then by (a),

$$
\begin{aligned}
\left\|T x_{n}-T x\right\| & =\left\|A x_{n} B x_{n}-A x B x\right\| \\
& =\left\|A x_{n} B x_{n}-A x B x_{n}\right\|+\left\|A x B x_{n}-A x B x\right\| \\
& \leq\left\|A x_{n}-A x\right\|\left\|B x_{n}\right\|+\|A x\|\left\|B x_{n}-B x\right\| \\
& \leq \phi\left(\left\|x_{n}-x\right\|\right)\|\overline{B(S)}\|+\|A x\|\left\|B x_{n}-B x\right\|
\end{aligned}
$$

Since $B: S \rightarrow X$ is compact, $\overline{B(S)}$ is compact and therfore $\|\overline{B(S)}\|<\infty$. Taking the limit as $n \rightarrow \infty$ in (3.1), $\lim _{n}\left\|T x_{n}-T x\right\|=0$ or $T x_{n} \rightarrow T x$ as $n \rightarrow \infty$. Hence $T$ is continuous on $S$.

Lemma 3.2. Let $S$ be a non-empty bounded subset of a Banach algebra $X$ and let $A, B: S \rightarrow X$ be two operators satisfying

(a) $A$ is D-Lipschitzician, and

(b) $B$ is continuous and compact.

Then for any subset $G$ of $S$,

$$
\alpha(T(G)) \leq M \phi(\alpha(G))
$$

where $T x=A x B x, x \in S$, and $M=\|B(S)\|$.

Proof. Let $x, y \in G$ be arbitrary, then we have

$$
\begin{aligned}
\|T x-T y\| & =\|A x B x-A y B y\| \\
& \leq\|A x-A y\|\|B y\|+\|A y\|\|B x-B y\| \\
& \leq\|B(G)\| \phi(\|x-y\|)+\|A y\|\|B x-B y\| .
\end{aligned}
$$

Now for any $x_{0} \in G$,

$$
\begin{aligned}
\|A y\| & \leq\left\|A x_{0}\right\|+\left\|A x_{0}-A y\right\| \\
& \leq\left\|A x_{0}\right\|+\phi\left(\left\|x_{0}-y\right\|\right) \\
& \leq\left\|A x_{0}\right\|+\phi(\operatorname{diam} G) \\
& \leq\left\|A x_{0}\right\|+\phi(\operatorname{diam} S) \quad(\because G \subset S) \\
& =\beta \\
& <\infty .
\end{aligned}
$$

Substituting this estimate in the inequality (3.3),

$$
\|T x-T y\| \leq\|B(S)\| \phi(\|x-y\|)+\beta\|B x-B y\|
$$


Let $\varepsilon>0$ be given, then there exist subsets $G_{1}, \ldots, G_{n}$ of $G$ such that $G=\bigcup_{i=1}^{n} G_{i}$ and $\operatorname{diam}\left(G_{i}\right)<\alpha(G)+\varepsilon$ for all $i=1, \ldots, n$. Since $B(G)$ is relatively compact, for any $n>0, \alpha(B(S))<\frac{\eta}{\beta}$ and there exist subsets $F_{1}, \ldots, F_{m}$ of $B(S)$ such that $B(S)=$ $\bigcup_{j=1}^{n} F_{j}$, or, equivalently $G \subset S=\bigcup_{j=1}^{n} B^{-1}\left(F_{j}\right)$ with $\operatorname{diam}\left(F_{j}\right)<\frac{\eta}{\beta} \forall j=1, \ldots, m$.

Therefore we have

$$
T(G)=\bigcup_{i, j} T\left(G_{i} \cap B^{-1}\left(F_{j}\right)\right)
$$

Since $\operatorname{diam}\left(G_{i}\right)<\alpha(G)+\varepsilon$ for all $i=1, \ldots, n$ we have

$$
\operatorname{diam}\left(G_{i} \cap B^{-1}\left(F_{j}\right)\right)<\alpha(G)+\varepsilon
$$

for all $i=1, \ldots, n$ and $j=1, \ldots, m$.

From (3.4) and (3.6) if follows that

$$
\operatorname{diam}\left(T\left(G_{i} \cap B^{-1}\left(F_{j}\right)\right)\right)<\max \{M \phi(r): r \in[\alpha(G), \alpha(G)+\varepsilon]\}+\eta
$$

for all $i=1, \ldots, n$ and $j=1, \ldots, m$.

Now

$$
\alpha(T(G))=\max _{i, j} \operatorname{diam}\left(T\left(G_{i} \cap B^{-1}\left(F_{j}\right)\right)\right),
$$

so we have from (3.7),

$$
\alpha(T(G))<\max \{M \phi(r): r \in[\alpha(G), \alpha(G)+\varepsilon]\}+\eta .
$$

As $\varepsilon$ and $\eta$ are arbitrary, we have

$$
\alpha(T(G)) \leq M \phi(\alpha(G))
$$

for all $G \subset S$. This completes the proof.

Theorem 3.1. Assume that all the conditions of Theorem 2.1 hold. Then the set $F i x(A B)=\{x \in S \mid A x B x=x\}$ is compact.

Proof. Define a mapping $T: S \rightarrow X$ by $T x=A x B x$. Then by Theorem 2.1, $\phi \neq F i x(A B)=$ Fix $(T)=\{x \in S \mid A x B x=x\}$. Moreover Fix $(A B)$ is a closed subset of $S$. To see this, let $\left\{x_{n}\right\}$ be a sequence in Fix $(T)$ converging to a point $x \in X$. We shall prove that $x \in \operatorname{Fix}(T)$. Now by Lemma $3.1, T$ is continuous, and so

$$
x=\lim _{n} x_{n}=\lim _{n} T x_{n}=T\left(\lim _{n} x_{n}\right)=T x
$$

As a result $x \in \operatorname{Fix}(T)$ and $F i x(A B)$ is a closed subset of $S$. If $F i x(T)$ is not compact, then by Lemma 3.2 with $G=F i x(A B)$, we obtain

$$
\alpha(F i x(T))=\alpha(T(F i x(T))) \leq M \phi(\alpha(F i x(T)))<\alpha(F i x(T))
$$


which is a contradiction. Hence Fix $(T)=F i x(A B)$ is compact. The proof is complete.

Theorem 3.2. Let $S$ be a closed convex and bounded subset of a separable Banach algebra $X$ and let $A(\omega), B(\omega): \Omega \times S \rightarrow X$ be two random operators satisfying for each $\omega \in \Omega$.

(a) $A(\omega)$ is D-Lipschitzician,

(b) $B(\omega)$ is completely continuous, and

(c) $A(\omega) x B(\omega) x \in S$ for each $x \in S$.

Then the random equation

$$
A(\omega) x B(\omega) x=x
$$

has a random solution whenever $M(\omega) \phi_{\omega}(r)<r, r>0$, for each $\omega \in \Omega$ where $M(\omega)=$ $\|B(\omega)(S)\|$.

Proof. Define an operator $T(\omega): \Omega \times S \rightarrow X$

$$
T(\omega) x=A(\omega) x B(\omega) x .
$$

Since $A(\omega)$ and $B(\omega)$ are random operators, $A(\omega) x$ and $B(\omega) x$ are $X$-valued random variables for all $x \in S$. As $X$ is a separable Banach algebra, the product of two $X$-valued random variables is again a random variable. Consequently $T(\omega) x=A(\omega) x B(\omega) x$ is a $X$-valued random variable. Hence $T(\omega)$ is a random operator on $S$. Moreover $T(\omega)$ is a continuous random operator on $S$ in view of Lemma 3.1.

Define a multi-valued map $F: \Omega \rightarrow 2^{S}$ by

$$
F(\omega)=\{x \in S \mid A(\omega) x B(\omega) x=x\} .
$$

Clearly by Theorem 2.1, $F(\omega)$ is non-empty for each $\omega \in \Omega$ and which is further compact in view of Theorem 3.2. To finish, it is enough to prove that $F$ is measurable on $\Omega$. Let $C$ be a closed subset of $X$. Denote

$$
L(C)=\cap_{n=1}^{\infty} \cup_{x_{i} \in C_{n}}\left\{\omega \in \Omega \mid\left\|x_{i}-A(\omega) x_{i} B(\omega) x_{i}\right\|<\frac{2}{n}\right\}
$$

where $C_{n}=\left\{x \in X \mid d(x, C)<\frac{1}{n}\right\}$ and $d(x, C)=\inf \{d(x, c) \mid c \in C\}$

Obviously $L(C)$ is a measurable subset of $\Omega$. We shall prove that $F^{-1}(C)=L(C)$. Obviously $F^{-1}(C) \subset L(C)$. Now proceeding with the arguments similar to that in the proof of Theorem 2.1 of Itoh [11], it is proved that $L(C) \subset F^{-1}(C)$. As a result $F^{-1}(C)=L(C) \in \mathcal{A}$. Hence $F$ is measurable on $\Omega$. Since $F(\omega)$ is compact, it has closed values for each $\omega \in \Omega$. Now an application of a theorem of Kuratowskii and Ryll-Nardzewski [14] yields that $F$ has a measurable selector, i.e. there is a measureable mapping $\xi: \Omega \rightarrow X$ such that $\xi(\omega) \in F(\omega)$ for all $\omega \in \Omega$. By definition of $F(\omega)$ which implies that $\xi(\omega)=A(\omega) \xi(\omega) B(\omega) \xi(\omega)$. This completes the proof.

Corollary 3.1. Let $S$ be a closed, convex and bounded subset of a separable Banach algebra $X$ and let $A(\omega), B(\omega): \Omega \times S \rightarrow X$ be two random operators satisfying for each $\omega \in \Omega$, 
(a) $A(\omega)$ is a Lipschitzician with a Lipschitz constant $\alpha(\omega)$.

(b) $B(\omega)$ is continuous and compact, and

(c) $A(\omega) x B(\omega) x \in S$ for each $x \in S$.

Then the random equation (3.8) has a random solution and the set of all such solutions is compact whenever $\alpha(\omega) M(\omega)<1$ for each $\omega \in \Omega$, where $M(\omega)=\|B(\omega)(S)\|$.

Recently Dhage and Regan [7] have obtained two companions of Leray-Schauder principle [8] involving the sum and product of two operators in a Banach space which are useful in the existence theory for nonlinear intregal equations of the mixed type. See also Dhage [6]. The slight generalizations of these nonlinear alternatives may be stated as follows.

Theorem 3.3. Let $U$ and $\bar{U}$ denote respectively the open bounded and closed bounded subsets of a Banach algebra $X$ containing the origin 0 and let $A: X \rightarrow X$ and $B: \bar{U} \rightarrow X$ be two operators satisfying

(a) A is D-Lipschitzician,

(b) $\left(\frac{I}{A}\right)^{-1}$ exists on $B(\bar{U})$,

(c) $B$ is completely continuous and

(d) $M \phi(r)<r, r>0$, where $M=\|B(\bar{U})\|$.

Then either

(i) the operator equation (2.5) has a solution in $\bar{U}$, or

(ii) the operator equation $\lambda A\left(\frac{x}{\lambda}\right) B x=x$ has a solution in $\partial \bar{U}$ for some $0<\lambda<l$ where $\partial \bar{U}$ is a boundary of $\bar{U}$.

Theorem 3.4. Let $U$ and $\bar{U}$ denote respectively the open and closed subsets of a Banach space $X$ containing the origin 0 and let $A: X \rightarrow X$ and $B: \bar{U} \rightarrow X$ be two operators such that

(a) $A$ is nonlinear contraction, and

(b) $B$ is completely continuous.

Then either

(i) the operator equation $A x+B x=x$ has a solution in $\bar{U}$, or

(ii) there exists an $u \in \partial \bar{U}$ such that $\lambda A\left(\frac{x}{\lambda}\right)+\lambda B u=u$ for some $\lambda \in(0,1)$ where $\partial \bar{U}$ is a boundary of $\bar{U}$.

Now we shall obtain the random versions of Theorems 3.3 and 3.4 which are again the companions of the random version of Leray-Schander principle.

Theorem 3.5. Let $U$ and $\bar{U}$ denote respectively the open bounded and closed bounded subsets of a separable Banach algebra $X$ containing the origin 0 , and let $A(\omega): \Omega \times X \rightarrow$ $X$ and $B(\omega): \Omega \times \bar{U} \rightarrow X$ be two random operators satisfying for each $\omega \in \Omega$,

(a) $A(\omega)$ is D-Lipschitzician, 
(b) $\left(\frac{I}{A}\right)^{-1}(\omega)$ exists on $B(\omega)(\bar{U})$

(c) $B(\omega)$ is completely continuous,

(d) $M(\omega) \phi(r)<r, r>0$, where $M(\omega)=\|B(\omega)(\bar{U})\|$, and

(e) there does not exist an $u \in \partial \bar{U}$ such that

$$
\lambda(\omega) A(\omega)\left(\frac{u}{\lambda(\omega)}\right) B(\omega) u=u \text { for any measurable } \lambda: \Omega \rightarrow \boldsymbol{R} \text { with } 0<\lambda(\omega)<1,
$$

where $\partial \bar{U}$ is a boundary of $\bar{U}$.

Then the random equation (3.8) has a random solution in $\bar{U}$.

Proof. The proof is similar to Theorem 3.2 and in this case we invoke Theorem 3.3 instead of Theorem 2.1 in the proof.

An interesting corollary to Theorem 3.5 in the applicable form is

Corollary 3.1. Let $B_{r}(0)$ and $\bar{B}_{r}(0)$ denote respectively the open and closed balls centered at the origin 0 of radius $r$ in a separable Banach Algebra $X$. Let $A(\omega): \Omega \times X \rightarrow$ $X$ and $B(\omega): \Omega \times \bar{B}_{r}(0) \rightarrow X$ be two random operators satisfying for each $\omega \in \Omega$,

(a) $A(\omega)$ is Lipschitzician with a Lipschitz constant $\alpha(\omega)$,

(b) $\left(\frac{I}{A}\right)(\omega)$ is well defined and one-to-one,

(c) $B(\omega)$ is continuous and compact.

(d) $\alpha(\omega) M(\omega)<1$, where $M(\omega)=\left\|B\left(\bar{B}_{r}(0)\right)\right\|$, and

(e) there does not exist an $u \in X$ with $\|u\|=r$ satisfying $\lambda A(\omega)\left(\frac{u}{\lambda}\right) B(\omega) u=u$ for any $0<\lambda<1$.

Then the random equation (3.8) has a random solution $\xi$ in $X$ with $\|\xi\| \leq r$.

Remark 3.1. Note that the random operator $\left(\frac{I}{A}\right)(\omega)$ is well defined if $A(\omega)$ is regular, i.e. $A(\omega)$ maps $X$ into the set of all invertible elements in $X$ for each $\omega \in \Omega$.

Theorem 3.6. Let $U$ and $\bar{U}$ denote respectively the open and closed subsets of a separable Banach space $X$ containing the origin 0 , and let $A(\omega): \Omega \times X \rightarrow X$ and $B(\omega): \Omega \times \bar{U} \rightarrow X$ be two random operators satisfying for each $\omega \in \Omega$,

(a) $A(\omega)$ is nonlinear contraction,

(b) $B(\omega)$ is completely continuous, and

(c) there does not exist an $u \in \partial \bar{U}$ such that

$\lambda(\omega) A(\omega)\left(\frac{u}{\lambda(\omega)}\right)+\lambda(\omega) B(\omega) u=u$ for any measurable $\lambda: \Omega \rightarrow \boldsymbol{R}$ with $\lambda \in(0,1)$.

where $\partial \bar{U}$ is a boundary of $\bar{U}$.

Then the random equation $A(\omega) x+B(\omega) x=x$ has a random solution in $U$.

An interesting corollary to Theorem 3.6 in its applicable form is 
Corollary 3.2. Let $B_{r}(0)$ and $\bar{B}_{r}(0)$ denote respectively the open and closed balls centered at the origin 0 of radius $r$, in a separable Banach space $X$. Let $A(\omega): \Omega \times X \rightarrow X$ and $B(\omega): \Omega \times \bar{B}_{r}(0) \rightarrow X$ be two random operators satisfying for each $\omega \in \Omega$,

(a) $A(\omega)$ is contraction,

(b) $B(\omega)$ is continuous and compact, and

(c) there does not exist an $u \in X$ with $\|u\|=r$ such that

$$
\lambda A(\omega)\left(\frac{u}{\lambda}\right)+\lambda B(\omega) u=u \text { for any } \lambda \in(0,1) .
$$

Then the random equation $A(\omega) x+B(\omega) x=x$ has a random solution $\xi$ in $X$ with $\|\xi\| \leq r$.

In the following section we shall apply our Theorem 3.2 to a certain class of nonlinear functional random intrgral equations of mixed type in a Banach algebra for proving the existence result under suitable conditions.

\section{Random Integral Equatioins}

Given a measurable space $(\Omega, \mathcal{A})$ and given a closed and bounded interval $J=[0,1]$ in $\boldsymbol{R}$, the set of all real numbers, consider the nonlinear functional random integral equation (in short FRIE) of mixed type,

$$
x(t, \omega)=\left[\int_{0}^{\mu(t)} f(s, x(\theta(s), \omega), \omega)\right]\left(q(t, \omega)+\int_{0}^{\sigma(t)} g(s, x(\eta(s), \omega), \omega) d s\right)
$$

for $t \in J$ and $\omega \in \Omega$; here $q: J \times \Omega \rightarrow \boldsymbol{R}$, and $f, g: J \times \boldsymbol{R} \times \Omega \rightarrow \boldsymbol{R}, \mu, \theta, \sigma, \eta: J \rightarrow J$.

The FRIE (4.1) is new to the literature and hence the results of this section are a new contribution to the theory of nonlinear random integral equations.

Let $M(J, \boldsymbol{R}), B(J, \boldsymbol{R}), B M(J, \boldsymbol{R})$ and $C(J, \boldsymbol{R})$ denote respectively the spaces of all measurable, bounded, bounded and measurable, and continuous real-valued functions on $J$. Notice that $C(J, \boldsymbol{R}) \subset B M(J, \boldsymbol{R}) \subset M(J, \boldsymbol{R})$.

We shall obtain the existence of the random solution to FRIE (4.1) in the space $B M(J, \boldsymbol{R})$ under suitable conditions. Define a norm $\|\cdot\|$ in $B M(J, \boldsymbol{R})$ by

$$
\|x\|=\max _{x \in J}|x(t)| .
$$

Clearly $B M(J, \boldsymbol{R})$ is a separable Banach algebra with this maximun norm. We need the following definition in the sequel.

Definition 4.1. A function $\beta: J \times \boldsymbol{R} \times \Omega \rightarrow \boldsymbol{R}$ is said to satisfy the condition of $L_{\omega}^{1}$-Caratheodory or is simply called $L_{\omega}^{1}$-Caratheodory if for each $\omega \in \Omega$.

(i) $t \rightarrow \beta(t, x, \omega)$ is measurable for each $\omega \in \Omega$.

(ii) $x \rightarrow \beta(t, x, \omega)$ is continuous almost everywhere for $t \in J$, and 
(iii) for given real number $k>0$, there exists a function $h_{k}: \Omega \rightarrow L^{1}(J, \boldsymbol{R})$ such that

$$
|\beta(t, x, \omega)| \leq h_{k}(t, \omega), \quad \text { a. e. } t \in J
$$

for all $x \in \boldsymbol{R}$ with $|x| \leq k$.

We consider the following hypotheses in the sequel.

$\left(\boldsymbol{A}_{\mathbf{0}}\right)$ The functions $\mu, \theta, \sigma, \eta: J \rightarrow J$ are continuous.

$\left(\boldsymbol{A}_{\mathbf{1}}\right)$ The function $\omega \rightarrow f(t, x, \omega)$ is measurable for all $t \in J$ and $x \in \boldsymbol{R}$.

$\left(\boldsymbol{A}_{\mathbf{2}}\right)$ The function $t \rightarrow f(t, x, \omega)$ is Reimann integrable for each $x \in \boldsymbol{R}$ and $\omega \in \Omega$.

$\left(\boldsymbol{A}_{\mathbf{3}}\right)$ There exists a function $\alpha: \Omega \rightarrow L^{1}(J, \boldsymbol{R})$ such that for each $\omega \in \Omega$.

$$
|f(t, x, \omega)-f(t, y, \omega)| \leq \alpha(t, \omega)|x-y|, \quad \text { a. e. } t \in J
$$

for all $x, y \in \boldsymbol{R}$.

$\left(\boldsymbol{A}_{\mathbf{4}}\right)$ There exists a function $\phi_{1}: \Omega \rightarrow L^{1}(J, \boldsymbol{R})$ such that for each $\omega \in \Omega$.

$$
|f(t, x, \omega)| \leq \phi_{1}(t, \omega), \quad \text { a. e. } t \in J
$$

for all $x \in \boldsymbol{R}$.

$\left(\boldsymbol{B}_{\mathbf{0}}\right)$ The function $q: \Omega \rightarrow C(J, \boldsymbol{R})$ is measurable.

$\left(\boldsymbol{B}_{\mathbf{1}}\right)$ the function $\omega \rightarrow g(t, x, \omega)$ is measurable for all $t \in J$ and $x \in \boldsymbol{R}$.

$\left(\boldsymbol{B}_{\mathbf{2}}\right) g(t, x, \omega)$ is $L_{\omega}^{1}$-Caratheodory.

$\left(\boldsymbol{B}_{3}\right)$ There exists a function $\phi_{2}: \Omega \rightarrow L^{1}(J, \boldsymbol{R})$ such that

$$
|g(t, x, \omega)| \leq \phi_{2}(t, \omega), \quad \text { a. e. } t \in J
$$

for all $\omega \in \Omega$ and $x \in \boldsymbol{R}$.

Theorem 4.1. Assume that the hypotheses $\left(\boldsymbol{A}_{\mathbf{0}}\right)-\left(\boldsymbol{A}_{\mathbf{4}}\right)$ and $\left(\boldsymbol{B}_{\mathbf{0}}\right)-\left(\boldsymbol{B}_{\mathbf{3}}\right)$ hold. Further if $\|\alpha(\omega)\|_{L^{1}} \times\left(\|q(\omega)\|+\left\|\phi_{2}(\omega)\right\|_{L^{1}}\right)<1$, for each $\omega \in \Omega$, then the FRIE (4.1) admits a random solution on $J$.

Proof. Let $X=B M(J, \boldsymbol{R})$. Define a subset $S$ of $X$ by

$$
S=\{x \in X \mid\|x\| \leq M(\omega) \forall \omega \in \Omega\}
$$

where $M(\omega)=\left\|\phi_{1}(\omega)\right\|_{L^{1}}\left(\|q(\omega)\|+\left\|\phi_{2}(\omega)\right\|_{L^{1}}\right)$.

Clearly $S$ is a closed convex and bounded subset of $X$. Consider the mapping $A(\omega), B(\omega): \Omega \times S \rightarrow X$ defined by

$$
A(\omega) x(t)=\int_{0}^{\sigma(t)} f(s, x(\theta(s), \omega), \omega) d s, \quad t \in J,
$$

and

$$
B(\omega) x(t)=q(t, \omega)+\int_{0}^{\sigma(t)} g(s, x(\eta(s), \omega)) d s, \quad t \in J .
$$


Since $\omega \rightarrow f(t, x, \omega)$ is measurable for all $t \in J$ and $x \in \boldsymbol{R}$ and the integral

$$
\int_{0}^{\mu(t)} f(s, x(\theta(s), \omega), \omega) d s
$$

is a limit of the finite sum of measurable functions, the function

$$
\omega \rightarrow \int_{0}^{\mu(t)} f(s, x(\theta(s), \omega), \omega) d s
$$

is measurable. As a result $A(\omega): \Omega \times S \rightarrow X$ is a random operator. Similarly the function

$$
\omega \rightarrow \int_{0}^{\sigma(t)} g(s, x(\eta(s), \omega)) d s
$$

is measurable. Since $X$ is separable, the sum of two measurable functions is measurable. Consequently the function

$$
\omega \rightarrow q(t, \omega)+\int_{0}^{\sigma(t)} g(s, x(\eta(s), \omega)) d s
$$

is measurable, and so $B(\omega): \Omega \times S \rightarrow X$ is a random operator.

Now the FRIE (4.1) is equivalent to the random equation $A(\omega) x B(\omega) x=x$. We shall show that the random operators $A(\omega)$ and $B(\omega)$ satisfy all the conditions of Corollary 3.1 on $S$.

Step I. First we show that $A(\omega)$ is a Lipschitzician, Let $x, y \in S$. Then by $\left(\boldsymbol{A}_{\mathbf{3}}\right)$,

$$
\begin{aligned}
|A(\omega) x(t)-B(\omega) x(t)| & =\left|\int_{0}^{\mu(t)} f(s, x(\theta(s), \omega), \omega) d s-\int_{0}^{\mu(t)} f(s, y(\theta(s), \omega)) d s\right| \\
& \leq \int_{0}^{\mu(t)}|f(s, x(\theta(s), \omega), \omega) d s-f(s, y(\theta(s), \omega), \omega) d s| \\
& \leq \int_{0}^{\mu(t)} \alpha(s, \omega)|x(\theta(s), \omega)-y(\theta(s), \omega)| d s \\
& \leq \int_{0}^{\mu(t)} \alpha(s, \omega)\|x(\omega)-y(\omega)\| d s \\
& =\|\alpha(\omega)\|_{L^{1}}\|x(\omega)-y(\omega)\| .
\end{aligned}
$$

Taking the maximum over $t$ in the above inequality, we obtain

$$
\|A(\omega) x-B(\omega) x\| \leq\|\alpha(\omega)\|_{L^{1}}\|x(\omega)-y(\omega)\|
$$

for all $x, y \in S$ and $\omega \in \Omega$. This shows that $A(\omega)$ is a Lipschitzician random operator on $S$ with Lipschitz constant $\|\alpha(\omega)\|_{L^{1}}$. 
Step II. Next we shall that $B(\omega)$ is a continuous and compact operator on $\Omega \times S$. Using $\left(\boldsymbol{B}_{\mathbf{2}}\right)$ and the dominated convergence theorems, it is shown by routine arguments that $B(\omega)$ is a continuous random operator on $S$. Let $\left\{x_{n}\right\}$ be a sequence in $S$. Then $\left\|x_{n}\right\| \leq M(\omega)$ for each $n \in \boldsymbol{N}$. Since $g(t, x, \omega)$ is $L_{\omega}^{1}$-Caratheodory, we have

$$
\begin{aligned}
B(\omega) x_{n}(t) & \leq|q(t, \omega)|+\int_{0}^{\sigma(t)}|g(s, x(\eta(s), \omega), \omega)| d s \\
& \leq|q(t, \omega)|+\int_{0}^{1} h_{M(\omega)}(s, \omega) d s \\
& \leq\|q(\omega)\|+\left\|h_{M(\omega)}(\omega)\right\|_{L^{1}}
\end{aligned}
$$

i.e. $\left\|B(\omega) x_{n}(t)\right\| \leq\|q(\omega)\|+\left\|h_{M(\omega)}(\omega)\right\|_{L^{1}}$

for all $\omega \in \Omega$, where $h_{M(\omega)}$ is a function given in the Definition 4.1(iii).

Therefore $\left\{B(\omega) x_{n}\right\}$ is a uniformly bounded sequence in $B(\omega)(S)$. Finally let $t, \tau \in J$ Then

$$
\begin{aligned}
\left|B(\omega) x_{n}(t)-B(\omega) x_{n}(\tau)\right| & \leq|q(t, \omega)-q(\tau, \omega)|+\left|\int_{\sigma(\tau)}^{\sigma(t)} g\left(s, x_{n}(s, \omega), \omega\right)\right| d s \\
& \leq|q(t, \omega)-q(\tau, \omega)|+\left|\int_{\sigma(\tau)}^{\sigma(t)} h_{M(\omega)}(s, \omega) d s\right| \\
& \leq|q(t, \omega)-q(\tau, \omega)|+|p(t, \omega)-p(\tau, \omega)|
\end{aligned}
$$

where $p(t, \omega)=\int_{0}^{\sigma(t)} h_{M(\omega)}(s, \omega) d s$.

Since $q(\cdot, \omega)$ and $p(\cdot, \omega)$ are continuous on the compact interval $J$ for each $\omega \in \Omega$, they are uniformly continuous. From (4.6) it follows that

$$
\left|B(\omega) x_{n}(t)-B(\omega) x_{n}(\tau)\right| \rightarrow 0 \quad \text { as } \quad t \rightarrow \tau .
$$

Hence $\left\{B(\omega) x_{n}\right\}$ is a equi-continuous set in $B(\omega)(S)$ for each $\omega \in \Omega$. Now an application of Arzela-Ascoli theorem yields that $B(\omega)(S)$ is compact for each $\omega \in \Omega$. As a result $B(\omega)$ is a compact random operator on $S$.

Step III. Notice

$$
\begin{aligned}
\|A(\omega) x B(\omega) x\| & \leq\|A(\omega) x\|\|B(\omega) x\| \\
& \leq\left(\max _{t \in J}|A(\omega) x(t)|\right)\left(\max _{t \in J}|B(\omega) x(t)|\right) \\
& \leq\left\|\phi_{1}(\omega)\right\|_{L^{1}}\left(\|q(\omega)\|+\left\|\phi_{2}(\omega)\right\|_{L^{1}}\right) \\
& =M(\omega)
\end{aligned}
$$

for each $x \in S$ and $\omega \in \Omega$, so $A(\omega) B(\omega) x \in S$ for each $x \in S$. 
Also we have

$$
\|\alpha(\omega)\|_{L^{1}}\|B(\omega)(S)\| \leq\|\alpha(\omega)\|_{L^{1}}\left(\|q(\omega)\|+\left\|\phi_{2}(\omega)\right\|_{L^{1}}\right)<1
$$

for each $\omega \in \Omega$. Thus all the conditions of Corollary 3.1 are satisfied an hence an application of it yields that the random equation $A(\omega) x B(\omega) x=x$ and consequently the FRIE (4.1) has a random solution $J$. This completes the proof.

As an application of the main existence result of this section, we prove the existence of the random solution of the following nonlinear functional random differential equation (in short FRDE) of neutral type,

$$
\left.\begin{array}{c}
\left(\frac{x(t, \omega)}{\int_{0}^{\mu(t)} f(s, x(\theta(s), \omega), \omega)}\right)^{\prime}=g(s, x(\eta(s), \omega), \omega), \text { a. e. } t \in J \\
x(0, \omega)=q(\omega)
\end{array}\right\}
$$

for all $\omega \in \Omega$, where $q: \Omega \rightarrow \boldsymbol{R}$ is meaurable, $f: J \times \boldsymbol{R} \times \Omega \rightarrow \boldsymbol{R}-\{0\}$ is continuous, $g: J \times \boldsymbol{R} \times \Omega \rightarrow \boldsymbol{R}$, and $\mu, \theta, \eta: J \rightarrow J$ are continuous with $\mu(t) \neq 0$, for all $t \in J$.

By the random solution of the FRDE (4.7) we mean a measurable function $x: \Omega \rightarrow$ $A C(J, \boldsymbol{R})$ that satisfies the equations in $(4.7)$, where $A C(J, \boldsymbol{R})$ is the space of all absolutely continuous real-valued functions on $J$.

Theorem 4.2. Assume that the hypotheses $\left(\boldsymbol{A}_{\mathbf{0}}\right)-\left(\boldsymbol{A}_{\mathbf{4}}\right)$ and $\left(\boldsymbol{B}_{\mathbf{0}}\right)-\left(\boldsymbol{B}_{\mathbf{3}}\right)$ hold. Further if $\|\alpha(\omega)\|_{L^{1}}\left(\|q(\omega)\|+\left\|\phi_{2}(\omega)\right\|_{L^{1}}\right)<1$, then the FRDE (4.7) admits a random solution on $J$.

Proof. The FRDE (4.7) is equivalent to the FRIE

$$
x(t, \omega)=\left[\int_{0}^{\mu(t)} f(s, x(\theta(s), \omega), \omega) d s\right]\left(b(\omega)+\int_{0}^{t} g(s, x(\eta(s), \omega), \omega) d s\right)
$$

for all $t \in J$ and $\omega \in \Omega$, where $b(\omega)=\frac{q(\omega)}{\int_{0}^{\mu(0)} f(s, x(\theta(s), \omega), \omega) d s}$

Now the desired conclusion follows by an application of Theorem 4.1 with $q(t, \omega)=$ $b(\omega)$ for all $t \in J$ and $\omega \in \Omega$, because $A C(J, \boldsymbol{R}) \subset B M(J, \boldsymbol{R})$. The proof is complete.

\section{Remarks and Conclusion}

The study of deterministic nonlinear integral equations of mixed type in a Banach algebra is initiated by the present author [J. Math. Phy. Sci. 25(1988), 603-611]. The study was motivated by the Chandrasekhar's H-equation in radiative heat transfer [4]. So some new fixed point theorems involving the product of two operators on a Banach algebra have been developed for this puropse. The work along this line is the most active area of research and the field is growing very rapidly. See Dhage [6], Dhage and Regan [7] 
and the reference therein. Here we point out that the process of radiative heat transfer usually involves the coefficient of heat transfer which depends upon the medium of heat conduction and so it would definitely play the significant role in the underlined transfer process. This unknown coefficient may be considered as the random parameter and in our opinion the indeterministic form of Chandrasekhar's H-equation is moe suitable to the needs of the specialists engaged in the study of accurate behaviour of said underlined phenomena. Hence the study of such equations in Banach algebras has got importance. In the present paper we have considered a much general form of the random integral equation than the random version of Changraskhar's H-equation in a Banach algebra and discussed only the existence of the solution, however, other aspects such as stability, continuance dependence and extremality of the solutions could also be discussed in the similar way. Again the random fixed point theorems developed in section III have nice applications to some other random integral equations in Banach algebras and some of the results in this direction will be reported elsewhere.

The author is thankful to the referee for some valuable suggestions.

\section{References}

[1] J. Banas and K. Goebel, Measures of Noncompactness in Banach Spaces, Marcel Dekker Inc. Now York, 1980.

[2] A. T. Bharucha Reid, Random Integral Equations, Academic Press, New York, 1972.

[3] D. W. Boyd and J. S. W. Wong, On nolinear contractions, Proc. Amer. Math. Soc. 20 (1969), 456-464.

[4] S. Chandrasekhar, Radiative Heat Transfer, Dover, New York, 1960.

[5] B. C. Dhage, On $\alpha$-condensing mappings in Banach algebras, The Math. Student 64(1994), 146-152.

[6] B. C. Dhage, On some nonlinear alternatives of Leray-Schaurder type with applications to functional integral equations, Indian J. Pure Appl. Math. 33(2002), 00-00.

[7] B. C. Dhage and D. O. Regan, A fixed-point theorem in Banach algebras with applications to functional integral equations, Functional Diff. Eqn. 7(2000), 259-267.

[8] J. Dugundji and A. Granas, Fixed Point Theory, Monographies Mathematyczne, PNW Warsaw, 1(1982).

[9] O. Hans, Random fixed point theorems, Transaction of the first prague Conference on information Theory, Statistical Decision Functions, Random Process, 105-125, 1957.

[10] C. J. Himmelbrg, Measurable relations, Fund. Math. 87(1957), 53-72.

[11] S. Itoh, Random fixed point theorems with applications to random differential equations in Banach spaces, J. Math. Anal. Appl. 67(1979), 261-273.

[12] M. A. Krasnoselskii, Topological Methods in the Theory of Nonlinear Integral Equations, Pergamon Press, 1964.

[13] K. Kuratowskii, Topology, Vol.1-PWN-Polish Scientific Publishers and Academic Press, Warsaw and New York, 1966.

[14] K. Kuratowskii and C. Ryll-Nardzewski, A general theorem on selectors, Bulll. Acad. Polon. Sciser. Sci. Math. Astrnom. Phy. 13(1965), 397-403. 
[15] N. S. Papageogiou, On the mesurable selection approach in random differential inclusion, fixed point theorey and optimizations, J. Math. Phy. Sci. 24(1990), 331-345.

[16] A. Mukherjea, Transformation aleatrotires separable, Theorem du point fixealeatoire, C. R. Acad. Sci. Paris. 263(1966), 393-395.

[17] B. L. S. Prakas Rao, Stochastic integral equations of mixed type II, J. Math. Phy. Sci. 7 (1973), 245-260.

[18] A. Spacek, Zufallige Gleichungen, Czechaslovak Math. J. 5(1955), 462-466.

[19] E. Zeidler,Nonlinear Functional Analysis and Its Applications: Part I, Springer Verlag, 1985.

"Kasubai", Gurukul Colony, Ahmedpur-413515, Dist: Latur, Maharashtra, India.

E-mail: bcd20012001@yahoo.co.in 